Original article

\title{
MICROBIOLOGICAL AND ANTIBACTERIAL RESISTANCE PROFILE IN CANINE OTITIS EXTERNA - A COMPARATIVE ANALYSIS
}

\author{
V. PETROV ${ }^{1}$, G. ZHELEV ${ }^{1}$, P. MARUTSOV ${ }^{1}$, K. KOEV $^{1}$, \\ S. GEORGIEVA ${ }^{2}$, I. TONEVA ${ }^{2} \&$ V. URUMOVA ${ }^{1}$ \\ ${ }^{1}$ Department of Veterinary Microbiology, Infectious and Parasitic Diseases, \\ ${ }^{2}$ Student; Faculty of Veterinary Medicine, Trakia University, Stara Zagora, Bulgaria
}

\section{Summary}

Petrov, V., G. Zhelev, P. Marutsov, K. Koev, S. Georgieva, I. Toneva \& V. Urumova, 2019. Microbiological and antibacterial resistance profile in canine otitis externa - a comparative analysis. Bulg. J. Vet. Med., 22, No 4, 447-456.

The aim of the present study was to compare the prevalence of microbial agents involved in canine otitis externa and their sensitivity to antibacterial drugs in two periods: 2007-2011 and 2013-2017. For 2013-2017, coagulase-positive staphylococci were the dominating bacterial species (186 isolates), followed by Pseudomonas aeruginosa (82 strains). The rate of isolated yeasts (mainly Malassezia pachydermatis) was substantially high (152 isolates). Compared to the earlier period (2007-2011) a tendency to more frequent occurrence of co-infections was noted $-61.7 \%$ and more than $80 \%$ of co-infections involved yeasts. Antibacterial resistance patterns showed a clear trend to increased resistance of coagulase-positive staphylococci and $\beta$-haemolytic streptococci to amoxicillin/clavulanic acid (42\% and 50\% respectively) and gentamicin $(29 \%, 40 \%)$. Increased resistance of $P$. aeruginosa was established to gentamicin (16\%) and amikacin (18\%). The prevalence of pseudomonads resistant to enrofloxacin was lower $(27 \%)$.

Key words: antimicrobial susceptibility, dog, microbial profile, otitis externa

\section{INTRODUCTION}

Otitis externa is acute or chronic inflammation of the ear auricle, horizontal and vertical ear canal and the outer tympanic membrane wall (Smith, 2015). The disease could be either primary (foreign bodies, ectoparasites etc.) or secondary following complications from ear canal stenosis, drooping ears, excessive hair in the ear canal, water in the ear canal, ob- structions, atopic dermatitis, allergic skin reactions to food, metabolic diseases, abnormal keratinisation, autoimmune diseases, injury during manipulations etc. Otitis externa is particularly common in canine pets with affection rates of 5-20\% (Angus, 2004; Greene, 2006; Lyskova et al., 2007; Terziev \& Urumova, 2018). 
The normal microflora of the ear canal involves mainly Staphylococcus spp. (coagulase-positive and coagulase-negative), $\beta$-haemolytic Streptococcus spp., Bacillus spp. (August, 1988, Lyskova et al., 2007). Also, the presence of Malassezia pachydermatis (M. pachydermatis), Microsporum canis and Otodectes cynoti was reported in ears without inflammation (Bornand, 1992; Hariharan et al., 2006; Aalbæk et al., 2010; Malayeri et al., 2010).

The commonest microbial pathogens associated to otitis externa are members of genera Staphylococcus, Streptococcus spp., Corynebacterium spp.; Pseudomonas aeruginosa ( $P$. aeruginosa), Proteus mirabilis ( $P$. mirabilis), Escherichia coli (E. coli) and Klebsiella pneumoniae (Gotthelf, 2004). Principal staphylococcal species reported to be involved in the etiology of the condition are coagulasepositive $S$. pseudintermedius or former $S$. intermedius (Devriese et al., 2009), S. aureus, S. schleiferi subs. coagulans and coagulase-negative $S$. epidermidis, $S$. schleiferi subs. schleiferi, $S$. simulans and S. saprophyticus (Lilenbaum et al., 2000; Hoekstra et al., 2002; Nagase et al., 2002; May et al., 2005). M. pachydermatis is also a common finding. Yeasts are widespread in animals having undergone continuous treatment with antibiotics. It should be stated that bacteria and yeasts are not primary pathogens of otitis externa but opportunistic species that replicated under favourable conditions created by another primary cause (Rosser, 2004; Miller et al., 2013).

The treatment of otitis externa in dogs still remains a great concern. Local antimicrobial therapy aimed at eradication of bacterial or yeast infections is commonly applied in small animal veterinary practices (Angus, 2004). The identification of the primary cause for ear inflammation is however essential for the success of the therapy (Jacobson, 2002). In most cases, antibacterial therapy is prescribed without identification of the microbial pathogen and its sensitivity to antimicrobial drugs. This approach is often inefficient when microorganisms are resistant to applied chemotherapeutics resulting in recurrence of otitis when the primary cause is not eliminated or due to resistant strains selection.

The aim of the present study was to investigate the changes in the prevalence of microbial agents of canine otitis externa and their sensitivity to antibacterial drugs in 2013-2017 in comparison to the period 2007-2011 (Petrov et al., 2013).

\section{MATERIALS AND METHODS}

The study was carried out between January 2013 and December 2017 in the microbiology lab of the Department of Veterinary Microbiology, Infectious and Parasitic Diseases, Faculty of Veterinary Medicine, Trakia University, Stara Zagora, Bulgaria.

\section{Animals and samples}

A total of 185 dogs with otitis externa were sampled. One hundred and twentyfour of dogs were with bilateral otitis, so a total of 248 samples were collected from both ears. The number of samples from dogs with unilateral otitis was 61. All 309 swab samples with secretion from the external ear canal were sent from private veterinary clinics for bacteriological examination and antibacterial sensitivity testing. They were stored in Amies transport medium under refrigeration conditions and transported to the lab by logistic companies.

The disease history in most cases comprised chronic course, empirical treat- 
ment without satisfactory outcome and frequent recurrencies.

\section{Microbiological examination}

Samples were parallelly cultured onto blood agar (base) (Merck, Germany) with 5\% sheep blood and McConkey agar (Merck, Germany). Cultures were incubated aerobically for $24-48 \mathrm{~h}$ at $37{ }^{\circ} \mathrm{C}$. For the mycological examination, the same samples were inoculated for 2-7 days on Sabouraud 4\% dextrose agar (Merck, Germany), supplemented with 0.4 $\mathrm{g} / \mathrm{L}$ chloramphenicol (>99.0\% HPLC, Fluka, China) and $0.5 \mathrm{~g} / \mathrm{L}$ actidione (cycloheximide, $>93.0 \%$ HPLC, Fluka, China), at $37^{\circ} \mathrm{C}$ and aerobic conditions. Staphylococcal isolates were identified as described in Bergey's Manual of Determinative Bacteriology (Holt et al., 1994) and Manual of Clinical Microbiology (Murray et al., 2003), on the basis of colony appearance, Gram staining, production of pigments, presence of haemolysis and biochemical behaviour (catalase, oxidase, coagulase production).

The sensitivity of bacterial isolates to antimicrobial drugs was tested by the disk diffusion methods and interpretation of results - by the Bauer-Kirby scoring sys- tem (Bauer et al., 1966), according to the Clinical and Laboratory Standards Institute (2013). The following disks loaded with antimicrobial substances were used: amoxicillin/clavulanic acid $(20 / 10 \mu \mathrm{g})$, cefquinome $(30 \mu \mathrm{g})$, gentamicin $(10 \mu \mathrm{g})$, tobramycin $(10 \mu \mathrm{g})$, amikacin $(30 \mu \mathrm{g})$, enrofloxacin $(5 \mu \mathrm{g})$, marbofloxacin (5 $\mu \mathrm{g})$, chloramphenicol (30 $\mu \mathrm{g})$, lincomycin/spectinomycin $(9 / 100 \mu \mathrm{g})$ and polymyxin B $(10 \mu \mathrm{g})$.

\section{Statistical analysis}

The determination of $95 \%$ confidence limits was performed with the help of statistical software GraphPad InStat v. 3.00 (GraphPad Software Inc., La Jolla, CA).

\section{RESULTS}

Negative microbiological finding was detected in 35 out of tested 309 swab samples. From the 274 samples with microbial growth, 505 strains were isolated (Table 1).

Monoinfections were established in $105(38.3 \%)$ of the 274 positive samples. Most commonly, the isolate was a coagu-

Table 1. Number and frequnecy of isolation of canine otitis externa pathogens for the period 20132017.

\begin{tabular}{lccc}
\hline Microbial pathogens & Number & $\begin{array}{c}\text { Isolation } \\
\text { rate }(\%)\end{array}$ & $\begin{array}{c}95 \% \text { confidence } \\
\text { limits }\end{array}$ \\
\hline Staphylococci (coagulase positive) & 186 & 36.83 & $33.1 \div 40.8$ \\
M. pachydermatis & 152 & 30.01 & $26.1 \div 33.4$ \\
P. aeruginosa & 82 & 16.24 & $13.4 \div 19.2$ \\
P. mirabilis & 18 & 3.56 & $2.5 \div 5.6$ \\
E. coli & 16 & 3.17 & $2.4 \div 5.8$ \\
Staphylococci (coagulase negative) & 15 & 2.97 & $2.1 \div 5.1$ \\
Streptococci ( $\beta$-haemolytic) & 15 & 2.97 & $2.1 \div 5.1$ \\
Candida spp. & 12 & 2.38 & $1.3 \div 2.7$ \\
Bacillus spp. & 5 & 0.99 & $0.3 \div 1.9$ \\
Corynebacterium spp. & 4 & 0.79 & $0.2 \div 1.6$ \\
\hline Total & 505 & 100.00 & \\
\hline
\end{tabular}




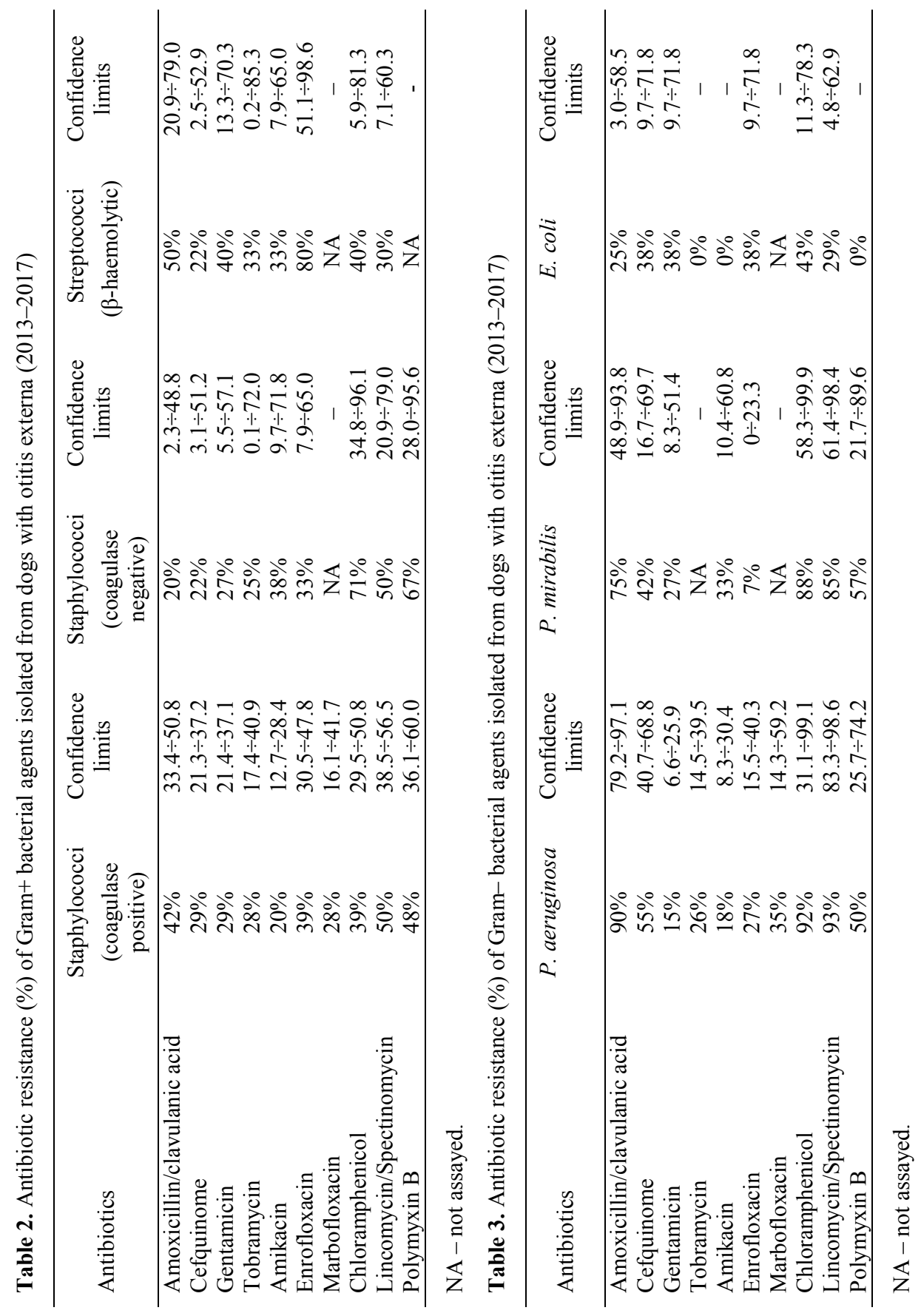



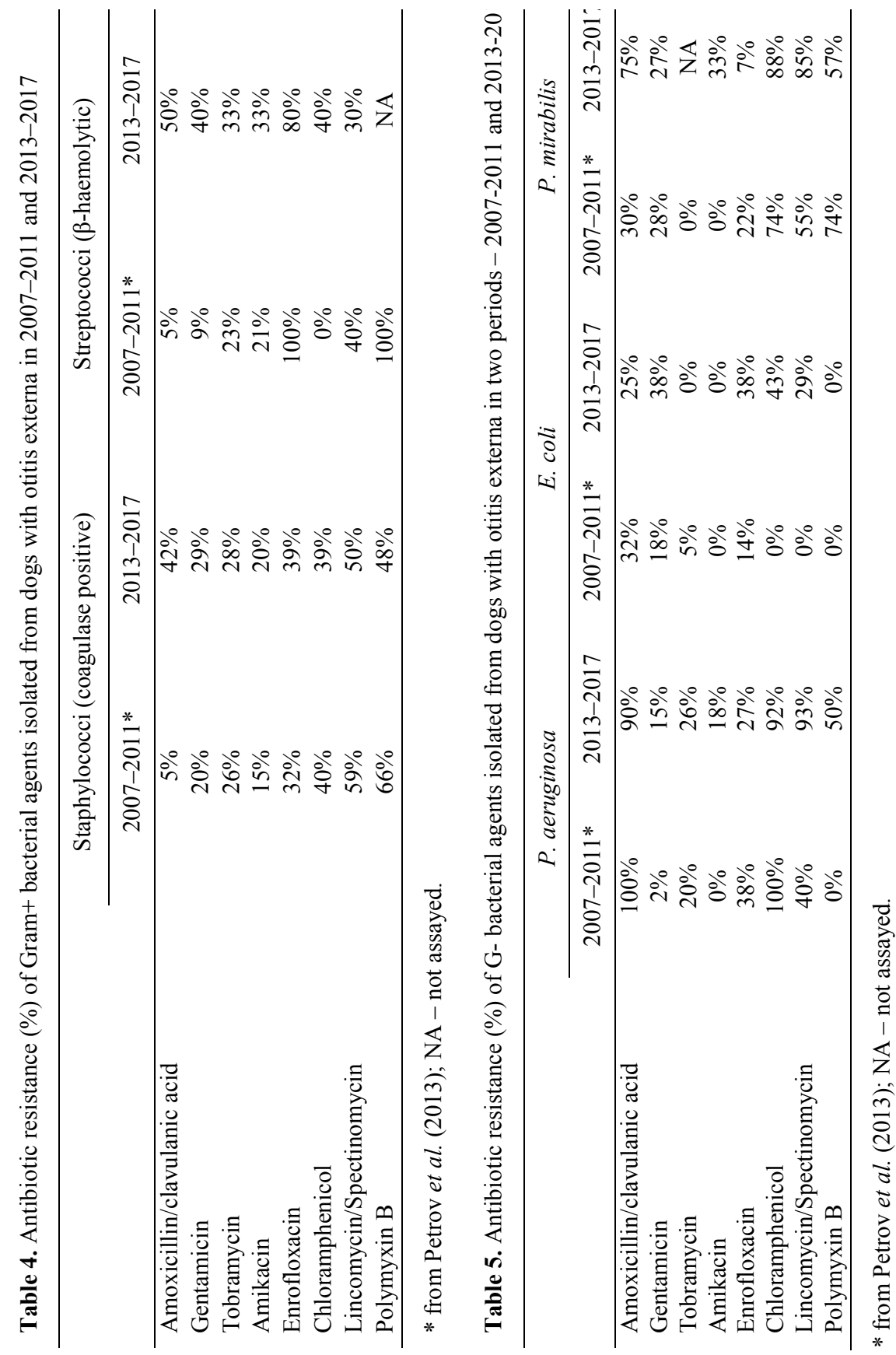

BJVM, 22, No 4 
lase-positive Staphylococcus and P. aeruginosa (39 and 31 cases, respectively), followed by $M$. pachydermatis (23 cases). Monoinfections induced by coagulasenegative staphylococci, P. mirabilis, Corynebacterium spp. and $\beta$-haemolytic streptococci were rare (in 5, 3, 3, 1 cases respectively). Co-infections were far more commonly seen (169 cases or $61.7 \%$ ), with main combinations between coagulase-positive staphylococci and M. pachydermatis (67 cases); coagulase-positive staphylococci and $P$. aeruginosa (11 cases); $M$. pachydermatis and $P$. aeruginosa (10 cases). In 44 samples, three microbial species were simultaneously isolated, also with predominance of associations between coagulase-positive staphylococci, M. pachydermatis and $P$. aeruginosa (15 cases) and coagulase-positive staphylococci, M. pachydermatis and $\beta$ haemolytic Streptococcus spp. (10 cases). From another ten samples, 4 microbial species were isolated at a time.

In 104 dogs, microbial findings were the same in left and right ears, so a total of 334 isolates including 92 yeasts strains were analysed. The antimicrobial sensitivity of 242 bacterial isolates was tested (Tables 2 and 3). Antibiotic resistance was a common finding. A very high resistance to amoxicillin with $\beta$-lactamase inhibitor was exhibited by coagulase-positive staphylococci. These bacteria were often resistant also to lincomycin/spectinomycin, polymyxin $\mathrm{B}$, chloramphenicol and fluoroquinolones. Staphylococci isolated from canine otitis externa were most sensitive to aminoglycosides and cefquinome.

As expected, the greatest resistance to antimicrobial drugs was demonstrated by pseudomonads, in particular against amoxicillin/clavulanic acid, lincomycin/spectinomycin and chloramphenicol. P. aerugi- nosa isolates had a relatively well preserved sensitivity to gentamicin and amikacin, although very resistant to enrofloxacin and marbofloxacin.

\section{DISCUSSION}

The comparison of data from 2013-2017 and those from the previous period 2007 2011 (Petrov et al., 2013) showed that staphylococci, M. pachydermatis and $P$. aeruginosa were the predominating microbial species isolated from dogs with otitis externa. In 2013-2017, coagulasepositive staphylococci were involved in more than $70 \%$ of cases. Marked increase of samples with yeast isolates (M. pachydermatis and Candida spp.) - over $65 \%$ and $P$. aeruginosa - 33\% was noted as compared to the previous period with prevalence rates of $40 \%$ and $17 \%$, respectively. This increase was probably due to the higher number of clinical samples from patients with chronic otitis, history of unsuccessful treatment or recurrent disease. Similar high prevalence of $M$. pachydermatis in dogs was reported by Crespo et al. (2002) in Spain, Nardoni et al. (2014) in Italy and Bardshiri et al. (2014) in Iran. Schick et al. (2007) have isolated $P$. aeruginosa in $53 \%$ of chronic canine otitis externa in line with our study.

The presence of $M$. pachydermatis monoinfection in 23 cases provided evidence for failure of antibiotic treatment prescribed without identification of the involved microbial agent. This is a commensal species on animal and human skin proliferating in the presence of favourable factors e.g. ear canal stenosis, excessive hairs, increased humidity and prolonged antibiotic treatment (Nardoni et al., 2014). In comparison to our previous research however (Petrov et al., 2013) the number of samples from which only yeasts were 
isolated has decreased. This could be probably attributed to the higher number of chronic otitis patients with polymicrobial associations. In this study, co-infections with participation of yeasts were present in almost $80 \%$ (140 out of 169 samples with more than one isolate), vs $50 \%$ (66 samples from 132 co-infections) in the previous study.

The analysis of sensitivity tests of bacterial isolates demonstrated high resistance of Gram+ bacteria to classical antimicrobial drugs: $\beta$-lactams and lincospectin. A special attention should be paid on the substantial increase of Gram+ bacteria resistant to the combination amoxicillin/clavulanic acid. Our data for sensitivity to amoxicillin/clavulanic acid are comparable to those of De Martino et al. (2016) and Saputra et al. (2017), but significantly higher that rates reported by Robaj et al. (2015); Metiner et al. (2015); Dziva et al. (2015) and Terziev \& Urumova (2018). The established increase of resistance of staphylococcal isolates to aminoglycosides, although not as pronounced, exceeded several times the rates from other studies. For instance Dziva et al. (2015) reported $17 \%$ resistance to gentamicin, De Martino et al. (2016) - 11\%, and Metiner et al. (2015) - only 2.5\%. Only $1.1 \%$ of staphylococcal strains isolated from dogs were not sensitive to amikacin in the research of Saputra et al. (2017). Similar to our resistance rates in staphylococci were reported by Robaj et al. (2015): more than $30 \%$.

The comparison of resistance of canine otitis externa bacterial isolates in the present study and the previous one (Petrov et al., 2013) showed that the resistance of Gram+ bacteria (coagulase positive staphylococci and $\beta$-haemolytic streptococci) to amoxicillin/clavulanic acid has increased 8-10 times: from $5 \%$ to $42 \%$ and $50 \%$. Resistance rates to the other tested antibiotics varied at a lesser extent, whereas the sensitivity of coagulasepositive staphylococci to polymyxin B was even increased from $34 \%$ to $52 \%$. A similar tendency: reduction of resistant isolates from $59 \%$ to $50 \%$ was observed with respect to the combination lincomycin/spectinomycin (Table 4).

The established higher resistance of streptococci to almost all tested antibiotics, in particular to amoxicillin/clavulanic acid, gentamicin and chloramphenicol as in line with the results of De Martino et al. (2016). It should be noted that streptococci are rarely isolated from canine otitis patients. Its occurrence rather in coinfections than in monoinfections suggest unsatisfactory outcome of antibiotic treatment undertaken without preliminary identification of the pathogen and its antimicrobial resistance profile.

With respect to Gram- bacteria, a more detailed analysis could be made for $P$. aeruginosa isolates because the number of $E$. coli and P. mirabilis strains was low. Expectedly, the percentage of pseudomonads resistant to chloramphenicol, amoxicillin/clavulanic acid and lincomycin/spectinomycin was very high. The detected resistant rates among Pseudomonas isolates in this study were higher than those reported by other researchers (Robaj et al., 2015; De Martino et al., 2016), but similar to those reported by Sutkevičiūte (2015) in Latvia.

The clear trend to increase in Pseudomonas strains resistant to the usual antimicrobial drugs used in clinical setting aminoglycosides, mainly gentamicin (Table 5) is of special concern for practicing veterinarians. On the other hand, a certain decline in the percentage of strains resistance to other used group of antimicrobials - fluoroquinolones (enrofloxacin in 
particular). The observed resistance in about one-third of $P$. aeruginosa isolates to enrofloxacin and marbofloxacin suggested a wrong approach to the therapy of Pseudomonas otitis in dogs resulting in selection of resistant strains. This pattern is probably similar in other countries as seen from reports about lower sensitivity of pseudomonads to enrofloxacin: $52 \%$ (Martin et al., 2000), 56.5\% (De Martino et al., 2016), only 35\% (Sutkevičiūtè, 2015).

A similar tendency was found out with respect to resistant $P$. mirabilis strains. This microbial species was outlined with the highest resistance to chloramphenicol, lincomycin/spectinomycin and amoxicillin/clavulanic acid. Proteus isolates were from chronic co-infections, mainly associated with $P$. aeruginosa and M. pachydermatis. Therefore, the resistance to these antimicrobial drugs had not a considerable effect on therapy efficacy as pseudomonads were also very resistant to them. The finding that almost one-third $P$. mirabilis strains were resistant to gentamicin and amikacin, particularly associations with pseudomonads for which these antibiotics are strategic, affirms the opinion on the wrong treatment approach to chronic canine otitis.

Among E. coli isolates, the resistance to gentamicin and enrofloxacin was almost twice increased. This fact suggests that the prescription of these antibacterial drugs without need is still a current practice and colibacteria are bacteria that evolve resistance very easily. This is not only a national problem. A study conducted in Australia has shown that $40 \%$ of veterinarians use fluoroquinolones empirically for therapy of canine otitis externa, and when cytology detected Gramnegative bacterial rods, their proportion increased to $61 \%$ (Hardefeldt et al.,
2017). This results in rapid evolution of antimicrobial resistant in bacterial strains, especially after prolonged treatments (Penna et al., 2010).

Similar results were found out with regard to resistance rates to chloramphenicol and lincomycin/spectinomycin - in the previous study of ours all E. coli isolates were sensitive to them while in the recent study, $30-40 \%$ of isolates were already resistant.

The yet preserved $100 \%$ sensitivity of $E$. coli isolates to amikacin, tobramycin and polymyxin B requires attentive use of these strategic antimicrobial drugs in the future.

\section{CONCLUSION}

The comparative analysis of prevalence of isolates from canine otitis demonstrated increased occurrence of commensal species $M$. pachydermatis and $P$. aeruginosa, along with increased resistance of bacteria to commonest antibacterial drugs. Therefore, the emphasis should be placed on the accurate etiological diagnosis and sensitivity tests to chemotherapeutics. This approach would allow for scientifically justified well-targeted antibiotic therapy.

\section{REFERENCES}

Aalbæk, B., D. A. Bemis, M. Schjærff, S. A. Kania, L. A. Frank \& L. Guardabassi, 2010. Coryneform bacteria associated with canine otitis externa. Veterinary Microbiology, 145, 292-298.

Angus, J. C., 2004. Otic cytology in health and disease. Veterinary Clinics of North America: Small Animal Practice, 34, 411-424.

August, J. R., 1988. Otitis externa: A disease of multifactorial etiology. Veterinary Clinics of North America: Small Animal Practice, 18, 731-742. 
Bardshiri, B., M. Tavana, S. Z. Peighambarzadeh, \& M. Abdolrahimi, 2014. Occurrence of Malassezia pachydermatis in the external ear canals of dogs with and without otitis externa. Walia Journal, 30, 24 26.

Bauer, A. W., W. M. Kirby, J. C. Sherris \& M. Turk, 1966. Antibiotic susceptibility testing by a standardized single disc method. American Journal of Clinical Pathology, 45, 493-496.

Bornand, V., 1992. Bacteriology and mycology of otitis externa in dogs. Schweizer Archiv fur Tierheilkunde, 134, 341-348.

Clinical and Laboratory Standards Institute, 2013. Performance standards for antimicrobial disk and dilution susceptibility tests for bacteria isolated from animals; approved standard. $4^{\text {th }}$ edn, CLSI, Wayne.

Crespo, M. J., M. L. Abarca \& F. J. Cabanes, 2002. Occurrence of Malassezia spp. in the external ear canals of dogs and cats with and without otitis externa. Medical Mycology, 40, 115-121.

De Martino, L., F. P. Nocera, K. Mallardo, S. Nizza, E. Masturzo, F. Fiorito, G. Lovane \& P. Catalanotti, 2016. An update on microbiological causes of canine otitis externa in Campania Region, Italy. Asian Pacific Journal of Tropical Biomedicine, 6, 384-389.

Devriese, L. A., K. Hermans, M. Baele, \& F. Haesebrouck, 2009. Staphylococcus pseudintermedius versus Staphylococcus intermedius. Veterinary Microbiology, 133, 206-207.

Dziva, F., C. Wint, T. Auguste, C. Heeraman, C. Dacon, P. Yu \& L. M. Koma, 2015. First identification of methicillin-resistant Staphylococcus pseudintermedius strains among coagulase-positive staphylococci isolated from dogs with otitis externa in Trinidad, West Indies. Infection Ecology \& Epidemiology, 5, 29170.

Greene, C. E., 2006. Otitis externa. In: Infectious Diseases of the Dogs and Cats, 3rd edn, ed C. E. Greene, Saunders Elsevier: St. Louis, MO, USA, pp. 815-823.
Hardefeldt, L. Y., S. Holloway, D. J. Trott, M. Shipstone, V. R. Barrs, R. Malik, M. Burrows, S. Armstrong, G. F. Browning \& M. Stevenson, 2017. Antimicrobial prescribing in dogs and cats in Australia: Results of the Australasian Infectious Disease Advisory Panel Survey. Journal of Veterinary Internal Medicine, 31, 1100-1107.

Hariharan, H., M. Coles, D. Poole, L. Lund \& R. Page, 2006. Update on antimicrobial susceptibilities of bacterial isolates from canine and feline otitis externa. The $\mathrm{Ca}$ nadian Veterinary Journal, 47, 253-255.

Hoekstra, K. A. \& R. J. L. Paulton, 2002. Clinical prevalence and antimicrobial susceptibility of Staphylococcus aureus and Staph. intermedius in dogs. Journal of Applied Microbiology, 93, 406-413.

Holt, J. G., N. R. Krieg, P. H. A. Sneathm, J. T. Staley \& S. T. Williams, 1994. Bergey's Manual of Determinative Bacteriology, $9^{\text {th }}$ edn, Williams and Williams, Baltimore, MD.

Gotthelf, L. N. 2004. Small Animal Ear Diseases: An Illustrated Guide. $2^{\text {nd }}$ edn, Elsevier Saunders, Philadelphia USA.

Jacobson, L. S., 2002. Diagnosis and medical treatment of otitis externa in the dog and cat. Journal of the South African Veterinary Association, 73, 162-170.

Lilenbaum, W., M. Veras, E. Blum \& G. N. Souza, 2000. Antimicrobial susceptibility of staphylococci isolated from otitis externa in dogs. Letters in Applied Microbiology, 31, 42-45.

Lyskova, P., M. Vydrzalova \& J. Mazurova, 2007. Identification and antimicrobial susceptibility of bacteria and yeasts isolated from healthy dogs and dogs with otitis externa. Transboundary and Emerging Diseases, 54, 559-563.

Malayeri, H. Z., S. Jamshidi \& T. Z. Salehi, 2010. Identification and antimicrobial susceptibility patterns of bacteria causing otitis externa in dogs. Veterinary Research Communications, 34, 435-444.

Martin, B. J. L., G. P. Lupiola, L. Z. Gonzalez $\&$ J. M. Tejedor, 2000. Antibacterial sus- 
ceptibility patterns of Pseudomonas strains isolated from chronic canine otitis externa. Zoonoses and Public Health, 47, 191-196.

May, E. R., K. A. Hnilica, L. A. Frank, R. D. Jones \& D. A. Bemis, 2005. Isolation of Staphylococcus schleiferi from healthy dogs and dogs with otitis, pyoderma, or both. Journal of the American Veterinary Medical Association, 227, 928-931.

Metiner, K., A. F. Bagcigil \& A. Ilgaz, 2015. Determination of the diversity and antibiotic resistance profiles of Staphylococcus species from dogs with otitis externa and examination of mecA gene occurrence. Veterinarni Medicina, 60, 261-267.

Miller, W. H., C. E. Griffin, K. L. Campbell \& G. H. Muller, 2013. Diseases of eyelids, claws, anal sacs, and ears. In: Muller and Kirk's Small Animal Dermatology, $7^{\text {th }}$ edn, Elsevier Health Sciences, pp. 724-774.

Murray, P. R., E. J. Baron, J. H. Jorgensen, M. A. Pfaller \& R. H. Yolken, 2003. Manual of Clinical Microbiology, $8^{\text {th }}$ edn, ASM Press, Washington, DC.

Nagase, N., A. Sasaki, K. Yamashita, A. Shimizu, Y. Wakita, S. Kitai \& J. Kawano, 2002. Isolation and species distribution of staphylococci from animal and human skin. Journal of Veterinary Medical Science, 64, 245-250.

Nardoni, S., V. V. Ebani, F. Fratini, R. Mannella, G. Pinferi, F. Mancianti, R. Finotello \& S. Permed, 2014. Malassezia, mites and bacteria in the external ear canal of dogs and cats with otitis externa. Slovenian Veterinary Research, 51, 113-118.

Penna, B., R. Varges, L. Medeiros, G. M. Martins, R. R. Martins \& W. Lilenbaum, 2010. Species distribution and antimicrobial susceptibility of staphylococci isolated from canine otitis externa. Veterinary Dermatology, 21, 292-296.

Petrov, V., G. Mihaylov, I. Tsachev, G. Zhelev, P. Marutsov \& K. Koev, 2013. Otitis externa in dogs: microbiology and antimicrobial susceptibility. Revue de Médecine Vétérinaire, 164, 18-22.
Robaj, A., D. Sylejmani \& A. Hamidi, 2015. Investigation and antimicrobial susceptibility of microbial agents of external otitis in dogs. Journal of Animal and Veterinary Advances, 14, 277-280.

Rosser Jr, E. J., 2004. Causes of otitis externa. Veterinary Clinics of North America: Small Animal Practice, 34, 459-468.

Saputra, S., D. Jordan, K. A. Worthing, J. M. Norris, H. S. Wong, R. Abraham, D. J. Trott \& S. Abraham, 2017. Antimicrobial resistance in coagulase-positive staphylococci isolated from companion animals in Australia: A one year study. PloS One, 12, 1-17.

Schick, A. E., J. C. Angus \& K. S. Coyner, 2007. Variability of laboratory identification and antibiotic susceptibility reporting of Pseudomonas spp. isolates from dogs with chronic otitis externa. Veterinary Dermatology, 18, 120-126.

Smith, F. W., 2015. Otitis externa and media. In: Blackwell's Five-Minute Veterinary Consult: Canine and Feline, John Wiley \& Sons.

Sutkevičiūtè, V., 2015. Pseudomonas aeruginosa išskyrimas iš šunų ausų ir atsparumo antimikrobinèms medžiagoms nustatymas. Doctoral dissertation, Lithuanian University of Health Sciences.

Terziev, G. \& V. Urumova, 2018. Retrospective study on the etiology and clinical signs of canine otitis. Comparative Clinical Pathology, 27, 7-12.

Paper received 30.01.2018; accepted for publication 10.05.2018

\section{Correspondence:}

Vladimir Petrov

Department of Veterinary Microbiology, Infectious and Parasitic Diseases,

Faculty of Veterinary Medicine,

Trakia University, 6000 Stara Zagora, Bulgaria, e-mail: vlado72@abv.bg 